\title{
Research on a 'drug-centred' approach to psychiatric drug treatment: assessing the impact of mental and behavioural alterations produced by psychiatric drugs
}

\author{
J. Moncrieff* \\ Reader in Critical and Social Psychiatry, Division of Psychiatry, University College London, London, UK
}

\begin{abstract}
Aims. This article explores an alternative understanding of how psychiatric drugs work that is referred to as the drugcentred model of drug action. Unlike the current disease-centred model, which suggests that psychiatric drugs work by correcting an underlying brain abnormality, the drug-centred model emphasises how psychiatric drugs affect mental states and behaviour by modifying normal brain processes. The alterations produced may impact on the emotional and behavioural problems that constitute the symptoms of mental disorders.
\end{abstract}

Methods. Arguments are put forward that justify the consideration of the drug-centred model. The research necessary to support the prescription of drugs according to such a model is explored.

Results. Evidence from neurochemistry and comparative drug trials do not confirm the disease-centred model of drug action. Since psychiatric drugs are recognised to have mind- and behaviour-altering properties, the drug-centred model constitutes a plausible alternative. The drug-centred model suggests that research is needed to identify all the alterations produced by various sorts of drugs, both acute and long term, and how these might interact with the symptoms and problems associated with different mental disorders. This requires detailed animal and volunteer studies and data from patients prescribed drug treatment long term, along with placebo-controlled and comparative trials that look at the overall impact of drug-induced alterations on well-being and functioning as well as symptoms. Research is also needed on alternative ways of fulfilling the function of drug treatment. The moral aspect of using drugs to modify behaviour rather than treat disease needs honest and transparent consideration.

Conclusions. It is hoped this discussion will encourage the psychiatric and pharmaceutical research community to provide more of the information that is required to use psychiatric drugs safely and effectively.

Received 12 August 2017; Accepted 11 September 2017; First published online 12 October 2017

Key words: Antidepressants, antipsychotics, drug mechanism of action, psychopharmacology, psychotropic drugs, research design and methods.

\section{Models of drug action}

Psychiatric drugs are currently understood to exert their beneficial effects by helping to normalise an underlying brain abnormality that is thought to be responsible for producing the symptoms of a particular mental disorder. Thus, antipsychotics are believed to reverse the pathology that produces psychotic symptoms or schizophrenia, antidepressants are believed to act on the biological processes that produce symptoms of depression, mood stabilisers are thought to help normalise the processes that produce abnormal mood swings and so on. The underlying pathology is sometimes proposed to consist of an imbalance in

* Address for correspondence: J. Moncrieff, Reader in Critical and Social Psychiatry, Division of Psychiatry, University College London, London, UK.

(Email: j.moncrieff@ucl.ac.uk) neurotransmitters or neurocircuitry (Hyman \& Nestler, 1996), but often it is not specified. This view of drug action is promoted by the pharmaceutical industry, whose websites frequently refer to the idea that psychiatric drugs work by 'balancing the chemicals naturally found in the brain' (Eli Lilly, 2006), and literature produced by professional organisations like the United Kingdom's Royal College of Psychiatrists and the American Psychiatric Association (American Psychiatric Association, 2005; Royal College of Psychiatrists, 2009).

Assumptions about drug action are rarely articulated, but this view can be set out in what I have called the 'disease-centred' model or theory of drug action (Table 1). The disease-centred model has been imported from general medicine, where most modern drugs are correctly understood in this way. Although most medical treatments do not reverse the original disease process, they act on the physiological processes that 
Table 1. Models of drug action

Disease-centred model

Drug-centred model

Drugs correct an abnormal brain state

Drugs as medical treatments

Beneficial effects arise from the drugs' action on the underlying disease process

Therapeutic effects can be distinguished from side effects
Drugs create an altered physiological state

Drugs as psychoactive substances

Beneficial effects are a consequence of being in an altered, drug-induced state

Some effects may be more useful than others produce symptoms. Thus, $\beta$ agonists help reverse airways obstruction in asthma and chemotherapeutic agents counteract the abnormal cell division that occurs in cancer. Analgaesics such as paracetamol and non-steroidal anti-inflammatory drugs also work in a disease-centred manner by acting on the physiological processes that produce pain.

The disease-centred model assumes that drugs exert their relevant effects only in people with a specific bodily abnormality or disease. The effects of drugs can therefore be meaningfully divided into the therapeutic effects, which are the effects on the disease process, and other effects, which are referred to as 'side effects'. The therapeutic effects will only be apparent in people who have the underlying pathology.

An alternative explanation for the effects of drugs in psychiatric disorders can be called the 'drug-centred' model of drug action. This model highlights that psychiatric drugs can be considered to be 'psychoactive' drugs in the sense that they are substances that cross the blood-brain barrier and affect brain functioning, thereby producing characteristic mental as well as physical alterations in anyone who ingests them (Moncrieff, 2008). There is no essential distinction, according to this view, between drugs used for psychiatric treatment and recreational psychoactive drugs like alcohol and cocaine. All psychoactive drugs produce altered physical and mental states, which can influence the way people think, feel and act, with different sorts of substances having different sorts of effects. The effects of recreational drugs are experienced as desirable by at least some people, but some drugs produce mental and physical changes that are generally disliked (e.g., antipsychotics and lithium). The drug-centred model suggests that it is these psychoactive properties that explain the changes seen when drugs are given to people with psychiatric problems. Drugs like benzodiazepines and alcohol, for example, reduce arousal and induce a usually pleasant state of calmness and relaxation. This state may be experienced as a relief for someone who is intensely anxious or agitated; but taking a drug like this does not return the individual to 'normal', or to their pre-symptom state. It is simply that the drug-induced state may be preferable to intense anxiety.

There are few examples of drugs working in a drugcentred way in modern medicine, but historically the psychoactive effects of alcohol were an important part of its analgaesic properties. Opiates also work partly through a drug-centred mechanism. Although they reduce pain directly by inhibiting the conduction of pain stimuli (a disease-centred action), they are psychoactive drugs that induce an artificial state of emotional indifference and detachment. People who have taken opiates for pain often say that they still have some pain, but do not care about it anymore.

According to a drug-centred model, therefore, psychiatric drugs produce a global state characterised by a range of physiological and psychological alterations. These alterations are likely to interact with the symptoms of mental disorders in ways that may sometimes be beneficial.

\section{Evidence on drug action}

Elsewhere I have documented how the disease-centred model of drug action evolved because of the vested professional, commercial and political interests it supported rather than the strength of scientific evidence. In fact, there is little evidence to suggest that any class of psychiatric drugs acts according to the diseasecentred model (Moncrieff \& Cohen, 2005; Moncrieff, 2008). Placebo-controlled trials do not distinguish whether drugs have a disease-centred or drug-centred action. They only indicate that drugs have different effects from an inert substance, the placebo.

Hypotheses about the neurochemical origins of psychiatric disorders that might support the diseasecentred model of drug action have not been substantiated. Antipsychotic drugs affect dopamine, among other neurotransmitters, but evidence for perturbations in the dopamine system that are specific to schizophrenia or psychosis, and independent of prior drug treatment, remains weak (Moncrieff, 2009; Kendler \& Schaffner, 2011). Studies of the dopamine 
content of post-mortem brains and dopamine metabolites are negative, for example. The increased concentration of dopamine $\mathrm{D}_{2}$ receptors, which was identified in brains of people with schizophrenia, transpired to be due to the effects of drug treatment. Recent studies report that indirect measures of dopamine activity are sometimes abnormal in people with acute psychosis. However, we know that dopamine is implicated in a range of functions including arousal, movement and stress that will confound its relations with any specific psychiatric disorder (Moncrieff, 2009). Moreover, the total number of drug-naïve participants in these studies is small.

Evidence on whether depression is caused by abnormalities of brain chemicals that might be reversed by drugs is even more contradictory. Studies of serotonin receptors, for example, show increased levels in depression in some studies, decreased levels in other studies and no difference in some (Moncrieff \& Cohen, 2006). There are claims that tryptophan depletion produces depression, but the research has involved people who had been previously treated with selective serotonin reuptake inhibitors (SSRIs), and studies with volunteers show no effects (Murphy et al. 2002). In 2001, the leading American textbook of psychiatry concluded that 'studies of serotonin function in depression suggest both hypofunction and hyperfunction' (Dubovsky et al. 2002). In 2013, psychopharmacologist, Stephen Stahl concluded: 'direct evidence for the monoamine hypothesis [of depression and mood disorders] is still largely lacking' (Stahl, 2013) (p. 262).

If the disease-centred model cannot be definitively supported, the drug-centred model of drug action has to be accepted as a possibility. No one disputes that psychiatric drugs alter normal mental functions, even if these 'psychoactive' effects have attracted little attention. It would be implausible to think these effects have no impact on the thoughts and behaviours that constitute the criteria for mental disorders.

\section{Using drugs according to a drug-centred model}

The drug-centred model suggests that drugs with psychoactive effects may affect the symptoms of mental disorders by virtue of their effects on mental states and behaviour in general. It is possible that in some situations, these interactions will be beneficial, at least from certain points of view. In other words, the behavioural adjustment produced by a drug may be judged to be preferable to the manifestations of the mental disorder (Breggin, 2008).

All sorts of drugs with sedative effects, for example, may be helpful in people with insomnia, anxiety and the distress or behavioural disturbance that accompanies acute psychosis and other situations. Antipsychotics produce a specific state of neurological inhibition, characterised by cognitive slowing, reduced initiative and motivation and emotional restriction that is not simply attributable to their (mostly) sedative effects (Breggin, 2008; Moncrieff et al. 2009). These effects are likely to reduce the intensity of emotional distress and psychotic thoughts, but may also impair global functioning.

Tricyclic antidepressants are strongly sedating, which suggests that they might be useful for symptoms of anxiety and insomnia across different disorders. SSRI antidepressants have more subtle psychoactive effects and are not profoundly sedating. They may produce a state of emotional restriction, which may reduce the intensity or salience of emotions. Whether these effects are really useful, whether they outweigh the negative aspects of taking mindand body-modifying chemicals, and whether they are superior to pharmacological and non-pharmacological alternatives still needs to be established, however.

\section{Research based on the drug-centred model}

In order to use and recommend drugs according to the drug-centred model, and to avoid iatrogenic harm, we need a whole body of research that has not yet been conducted due to the dominance of the disease-centred model over psychopharmacological research. We need more comprehensive data about the changes that drugs produce in mental and physical states, and then we need research to explore how those changes might interact with the manifestations of mental health problems and impact on the wider lives of people taking them. Crucially we need data that match the duration of time for which people typically take these drugs. We also need to consider the moral aspects of using drugs that modify emotional states and behaviour.

\section{Acute psychoactive and physical effects}

Detailed studies with animals and volunteers are needed to establish the acute alterations different drugs produce. Animal studies need to measure changes in spontaneous behaviour and tests of cognitive performance. Volunteer studies can provide additional information about the subjective alterations produced by particular drugs including changes in emotions, sensations and cognitive experiences. These alterations need to be reported in descriptive, phenomenological terms that can capture the unusual and often unfamiliar nature of drug-induced states, 
just as early users described the experience of taking psychedelic drugs (Huxley, 1954). Human volunteers should also be asked to reflect on the experience once the drug's effects have worn off, since it may be difficult to articulate this fully while under the influence of the substance. Despite the fact that volunteer studies are required as part of drug-licensing procedures, this sort of rich and detailed information has not traditionally been considered of interest. Only one recent scientific study conducted in the 1990s compared results of psychological tests and subjective experiences in volunteers randomised to take a single dose of the antipsychotic droperidol, a benzodiazepine or a placebo. Participants who took the antipsychotic could only describe certain aspects of the drug experience after the effects had worn off (Healy \& Farquhar, 1998).

Psychoactive drugs also produce physical or bodily changes, which may be linked with mental changes. Indeed, these drugs are best understood as producing altered global states that have both physical and mental components. The sedation produced by benzodiazepines, and the stimulation produced by stimulants, for example, are both simultaneously physical and mental experiences. The sedation and emotional indifference produced by antipsychotics like olanzapine may be related to their metabolic effects (Moncrieff et al. 2009). Monitoring how drugs affect different bodily systems is important to understand the full nature of a drug's effects and to explore correlations between physical changes and mental alterations.

\section{Long-term changes}

Long-term use of a drug has both predictable and unpredictable effects on the body. The alterations that occur if a drug is taken repeatedly may be reduced, the phenomena known as tolerance, or alternatively enhanced, which is known as sensitisation. Tolerance appears to be the more common of these scenarios, due to bodily adaptations that counteract some of a drug's effects, but the phenomena have not been adequately investigated for psychiatric drugs (as opposed to recreational drugs) and mechanisms remain unclear $(\mathrm{Li}, 2016)$. However, some studies suggest that olanzapine and haloperidol progressively lose their behavioural effects in animals, which is reflected in adaptations of the dopamine system (Samaha et al. 2007). Tolerance to benzodiazepine effects is also recognised, although the precise mechanism is not known (Vinkers \& Olivier, 2012).

Psychiatric medications are usually prescribed for long periods of time, and therefore it is essential to have good information about all the potential alterations and complications that occur with longterm treatment in order to make judgements about the cost-benefit ratio of using a particular drug. A host of physical complications are documented for every class of drug, but there is often little information about the prevalence, severity, reversibility and underlying mechanism of such effects. Moreover, less tangible effects are often overlooked in the mainstream literature, even when they are widely reported by users such as the emotion-numbing effects of antipsychotics and antidepressants, and post-SSRI sexual dysfunction.

The effects of long-term treatment have been underresearched for a number of reasons. Most obvious are the difficulties of conducting long-term studies in animals or volunteers. Studies with patients taking long-term medication are sometimes thought to be unreliable because of the difficulty of distinguishing some drug-induced effects from symptoms of the underlying conditions. The disease-centred model, moreover, focuses attention on a drug's effects on the presumed disease process, with other effects relegated to the status of 'side effects'. These might be elicited using a single list, but are rarely considered worthy of detailed attention. These difficulties can result in delayed recognition of serious and sometimes irreversible adverse effects, which is illustrated by the history of tardive dyskinesia (Moncrieff, 2013) and more recently by the case of post-SSRI sexual dysfunction (Bahrick, 2008).

Again, animal studies are required to identify changes in animal behaviour and performance following long-term treatment, as well as bodily changes. The importance of such studies has been underlined recently by the identification of brain volume reductions in rats and monkeys following long-term antipsychotic administration (Dorph-Petersen et al. 2005; Vernon et al. 2011), changes which are also apparent in patients (Ho et al. 2003).

Data also need to be collected systematically from people who are on long-term treatment for mental health problems, given the difficulties of conducting long-term volunteer studies. Looking for common themes and consistencies across different diagnostic groups will help enhance the validity of such data. As with acute effects, it is important to pay attention to the retrospective reflections of people who have discontinued medication since people may not be aware of the full effects of medication while they are under the influence of it. Although this sort of data has been dismissed in the past, several new initiatives have been designed to capture and explore patient reports in more detail (RxISK, 2017). A comparative study found such sites identify a similar profile of effects as those presented on professionally controlled 
websites but provide richer detail (Hughes \& Cohen, 2011).

\section{Withdrawal effects}

Along with acute alterations and longer term changes, patients and clinicians need to know what effects might occur following discontinuation of a drug. It is now widely acknowledged that withdrawal effects can occur following cessation of all sorts of drugs prescribed for mental health problems. However, there are little preclinical data on the prevalence or nature of withdrawal effects. Animal studies that assess withdrawal effects should be routine and more comprehensive, and long-term data need to be collected from patients who have discontinued medication. Although withdrawal effects are reported in the literature, there remain few studies that set out to investigate these effects in a comprehensive and systematic manner. It is also important to establish the course of changes following withdrawal. Some patients report protracted symptoms following withdrawal of benzodiazepines and antidepressants, for example (Fava et al. 2015), and so far we are unable to clarify whether withdrawal-related changes normalise over time in everyone or not.

\section{Neurotransmitter research}

Data on the neurochemical correlates of the behavioural, emotional and cognitive changes produced by different drugs are interesting, but the situation is likely to be more complex than has so far been presented. It is rarely appreciated that we do not have a good understanding of the full functions of any neurotransmitter system, and it is likely that their effects are all deeply interconnected.

Moreover, most drugs affect a range of neurochemical systems in a variety of ways. Although interest has centred on the action of antipsychotic drugs on the dopamine and serotonin systems, for example, these drugs also have varying effects on the noradrenergic and histaminergic systems in ways that are likely to be significant in the behavioural alterations they produce for some agents.

The difficulty of linking specific neurochemicals with manifest behaviour means that we have to start, as stressed above, with detailed descriptions of the changes that drugs produce in living organisms, both animals and humans. With more sophisticated techniques, we may one day be able to link these with specific neurochemical processes. Alternatively, it may be the case that the complexity of inter-relations between brain states and behaviour will always exceed our ability to describe it.

\section{Interaction of psychoactive effects and mental symptoms}

Once the acute and longer term alterations produced by a particular drug have been established, it is possible to start evaluating how those alterations might interact with the symptoms of various mental disorders, and whether any beneficial or desirable consequences might result.

First, we need to know how the alterations produced by a particular drug affect specific symptoms. Is the characteristic state of neurosuppression produced by antipsychotics effective at reducing the intensity of psychotic experiences and the disturbed behaviour that often accompanies these, for example? Existing placebo-controlled trials provide some information about this, but we also need comparative trials to demonstrate whether the particular effects of antipsychotics are superior to the effects of other drugs with psychoactive effects that might plausibly be useful, including other sedative agents like benzodiazepines. Similarly, we might explore whether the emotional numbing produced by some antidepressants impacts on depressed mood, anxiety and other symptoms. Again, we need to clarify whether this effect is specific to a certain class of drugs, or whether similar effects are obtained with other psychoactive agents.

The role of placebo effects is important in research based on a drug-centred model of drug action, as it is in current research. However, by highlighting the fact that psychiatric drugs produce noticeable physical and mental alterations and can therefore often be distinguished from an inert placebo, the drug-centred model suggests that use of inert placebos may not adequately control for the effects of expectations. Use of active placebos may be useful, but unless the alterations experienced with the 'active placebo' drug are identical to those produced by the agent being investigated, it is not possible to rule out expectation effects definitively. There is also the problem that any substance that is found to have effects is often designated as a specific agent. A variety of psychoactive substances have been found to be modestly superior to an inert placebo in depression trials, for example, some of which are designated as antidepressants and some are not. The fact that these drugs, including those that are considered as antidepressants, have such diverse pharmacological and subjective effects suggests that they may be acting as amplified placebos, with the psychoactive effects facilitating placebo or expectation effects.

Second, as well as establishing effects on symptoms, we need to evaluate a drug's effects on other aspects of well-being, using both subjective and objective 
measures. Antipsychotics may have useful effects on psychotic experiences, for example, but this may not necessarily translate into overall benefits on quality of life or functioning. We know that antipsychotics cause impairment of normal neuropsychological functioning in animals and volunteers, and that their effects are often experienced as highly disagreeable by users (Moncrieff, 2013). Similarly, the effects of antidepressants and other drugs on emotional experience may, or may not, translate into wider benefits.

Third, we need to establish whether any identified benefits outweigh adverse effects, bearing in mind that some adverse effects take time to emerge. The concepts of 'number needed to treat' and 'number needed to harm' represent attempts to balance benefits and harms in this way, but we need data that evaluate broader outcomes than just symptom improvement, consider all potential adverse effects, and compare different types of drugs.

All these factors have to be re-evaluated throughout the course of long-term treatment if this is to be instituted. Benefits of long-term treatment may be more difficult to establish, because of the difficulty of conducting long-term trials and the confounding effects of discontinuation-related adverse effects in trials that involve discontinuation of established long-term treatment (Moncrieff, 2006). It is vitally important, however, that there are more data on long-term costs and benefits of drug treatment in view of the fact that longterm treatment is associated with increasing burden and probability of adverse effects, and that benefits of drugs may reduce with continuing use due to natural remission or improvement of symptoms and pharmacological tolerance.

\section{Alternatives to drug treatment}

When we have clarified what drug-induced alterations can achieve, we are in a position to compare these with alternative strategies. For this, it is important to understand the role that drug treatment plays, or is intended to play, in an individual's life. The desired purposes of treatment may be obscured, however, if people believe they are taking a drug to treat an underlying disease. Where it appears that drug treatment is desired to reduce the intensity of negative emotions, for example, we need data on comparative outcomes of other ways of managing emotions, such as exercise, therapy or engaging in new activities. We should also consider how drug treatment might hamper the development and utilisation of other strategies for regulating emotions, either through direct effects on mental functioning, or by suggesting that symptoms are beyond reach by other means. Research into ways of helping people to manage psychotic symptoms that might avoid or reduce the need for antipsychotic treatment would also be useful.

\section{Moral considerations}

The sort of behavioural and emotional changes that drugs produce in different situations may be a scientific question, but the drug-centred model highlights that there is also a moral question about whether these changes are desirable or not. In many cases, there is consensus about the value of such changes, but there are situations in which different parties evaluate the desirability of drug-induced changes differently. The tranquilisation produced by antipsychotics in an acutely aggressive patient will be welcomed by onlookers (including staff), for example, but is often intensely unpleasant for the patient. Indeed, one study found that recovered patients who had experienced rapid tranquilisation said they would have preferred physical restraint (Schmeid \& Ernst, 1983). Similarly, the behavioural changes produced by longterm antipsychotic treatment may be valued by professionals and carers, but disliked by the patient.

For this reason, it is important to clarify the exact purpose of drug treatment in every situation, and whose perspective this purpose serves. Where the primary aim of treatment is to make someone's behaviour less risky or inconvenient to other people, careful moral and legal consideration is required to consider whether this is a legitimate goal or not.

\section{Implications for prescribing practice}

A drug-centred approach changes the nature of prescribing, and relationships between patients and prescribers. Under the disease-centred model, prescribing is driven primarily by diagnosis and theories about the aetiology of the hypothetical underlying condition. A drug-centred approach, in contrast, focuses on making judgements about when drug-induced mental and behavioural modifications might be useful. Research data can inform doctor and patient about the nature of drug-induced effects and their impact on various outcomes, but the utility of a drug can only be established by assessing each individual's experience of the whole range of drug-induced effects in their own particular situation. It is important to monitor the consequences of drug-induced alterations on all areas of mental and physical functioning, therefore, including the identification of subtle changes (such as changes to emotional responsiveness) which may not be routine.

The drug-centred model therefore advocates a collaborative form of self-medication, in which psychiatrists act as reservoirs of information on drugs' 
psychoactive and physical effects, help to explore the likely overall impact and limitations of drug treatment and consider alternative, non-drug-based approaches.

The drug-centred model can support psychiatrists to rationalise and reduce medication when it is ineffective or harmful and to resist inappropriate prescribing. Doctors often feel an expectation to prescribe, with some patients viewing a prescription as an acknowledgement of their suffering and non-prescription as a denial (Pellegrino, 1976). The disease-centred model can reinforce these expectations, and patients may become trapped in a cycle of ever-increasing medication, because the social and personal drivers of symptoms are obscured. The drug-centred model, in contrast, offers a way of exploring the potential benefits of drug therapy, without raising expectations that it is an essential part of the solution, and of considering non-pharmacological ways of fulfilling the function that medication is intended to perform.

\section{Conclusions}

This article argues that out current assumptions about the nature of drug action are not supported by evidence. An alternative, 'drug-centred' model of drug action is proposed, which changes the way we understand what psychiatric drugs do when they are used to treat a mental disorder. The drug-centred model emphasises that psychiatric drugs are psychoactive substances that change the normal state of the body and brain. The modifications in mental state and behaviour that result may reduce the manifestations of some mental disorder, but there is more to consider and to evaluate whether these changes are worthwhile. The article presents the types of research that we need to produce comprehensive information about all the alterations that different drugs produce and how these might interact with the symptoms of mental disorders, in order to be able to weigh up the pros and cons of using them safely and effectively.

Currently much of this information is lacking. Clinicians prescribe drugs for all sorts of mental disorders, often for long periods of time, with inadequate knowledge about all the consequences of doing so. It is beholden on the psychiatric and psychopharmacology research community to investigate the alterations produced by psychiatric drugs more thoroughly, so that clinicians and patients can make better informed decisions about the value of using drug treatment in various situations.

\section{Financial Support}

This research received no specific grant from any funding agency, commercial or not-for-profit sectors.

\section{Conflict of Interest}

The author has no conflicting financial interests. She is the co-chair person of the Critical Psychiatry Network, an international group of psychiatrists who challenge the dominance of the biomedical approach in psychiatry.

\section{Ethical Standard}

The authors assert that all procedures contributing to this work comply with the ethical standards of the relevant national and institutional committees on human experimentation and with the Helsinki Declaration of 1975, as revised in 2008.

\section{References}

American Psychiatric Association (2005). Lets talk facts about depression. Retrieved 13 February 2006 from http:// www.healthyminds.org/multimedia/depression.pdf.

Bahrick A (2008). Persistence of sexual dysfunction side effects after discontinuation of antidepressant medications: emerging evidence. The Open Psychology Journal 1, 42-50.

Breggin P (2008). Brain-Disabling Treatments in Psychiatry. Springer Publishing Company: New York.

Dorph-Petersen KA, Pierri JN, Perel JM, Sun Z, Sampson AR, Lewis DA (2005). The influence of chronic exposure to antipsychotic medications on brain size before and after tissue fixation: a comparison of haloperidol and olanzapine in macaque monkeys. Neuropsychopharmacology 30, 1649-1661.

Dubovsky SL, Davies R, Dubovsky AN (2002). Mood disorders. In Textbook of Clinical Psychiatry, 4th ed. (ed. RE Hales and SC Yudofsky), pp. 439-542. American Psychiatric Publishing: Washington, DC.

Eli Lilly (2006). Zyprexa website. Retrieved 10 November 07 from http://www.zyprexa.com/patient/howworks.jsp/.

Fava GA, Gatti A, Belaise C, Guidi J, Offidani E (2015). Withdrawal symptoms after selective serotonin reuptake inhibitor discontinuation: a systematic review.

Psychotherapy \& Psychosomatics 84, 72-81.

Healy D, Farquhar G (1998). Immediate effects of droperidol. Human Psychopharmacology 13, 113-120.

Ho BC, Andreasen NC, Nopoulos P, Arndt S, Magnotta V, Flaum M (2003). Progressive structural brain abnormalities and their relationship to clinical outcome: a longitudinal magnetic resonance imaging study early in schizophrenia. Archives of General Psychiatry 60, 585-594.

Hughes S, Cohen D (2011). Can online consumers contribute to drug knowledge? A mixed-methods comparison of consumer-generated and professionally controlled psychotropic medication information on the internet. Journal of Medical Internet Research 13, e53.

Huxley A (1954). Doors of Perception. Chatto \& Windus: London.

Hyman SE, Nestler EJ (1996). Initiation and adaptation: a paradigm for understanding psychotropic drug action. American Journal of Psychiatry 153, 151-162. 
Kendler KS, Schaffner KF (2011). The dopamine hypothesis of schizophrenia: an historical and philosophical analysis. Philosophy, Psychiatry \& Psychology 18, 41-63.

Li M (2016). Antipsychotic-induced sensitization and tolerance: behavioral characteristics, developmental impacts, and neurobiological mechanisms. Journal of Psychopharmacology 30, 749-770.

Moncrieff J (2006). Why is it so difficult to stop psychiatric drug treatment? It may be nothing to do with the original problem. Medical Hypotheses 67, 517-523.

Moncrieff J (2008). The Myth of the Chemical Cure: A Critique of Psychiatric Drug Treatment. Palgrave Macmillan:

Basingstoke, Hampshire, UK.

Moncrieff J (2009). A critique of the dopamine hypothesis of schizophrenia and psychosis. Harvard Review of Psychiatry 17, 214-225.

Moncrieff J (2013). The Bitterest Pills: The Troubling Story of Antipsychotic Drugs. Palgrave Macmillan: London.

Moncrieff J, Cohen D (2005). Rethinking models of psychotropic drug action. Psychotherapy \& Psychosomatics 74, 145-153.

Moncrieff J, Cohen D (2006). Do antidepressants cure or create abnormal brain states? PLoS Medicine 3, e240.

Moncrieff J, Cohen D, Mason JP (2009). The subjective experience of taking antipsychotic medication: a content analysis of Internet data. Acta Psychiatrica Scandinavica 120, 102-111.

Murphy FC, Smith KA, Cowen PJ, Robbins TW, Sahakian BJ (2002). The effects of tryptophan depletion on cognitive and affective processing in healthy volunteers.

Psychopharmacology (Berlin) 163, 42-53.

Pellegrino ED (1976). Prescribing and drug ingestion symbols and substances. Drug Intelligence \& Clinical Pharmacy 10, 624-630.

Royal College of Psychiatrists (2009). Antidepressants. Royal College of Psychiatrists: London. Retrieved 11 August 2017 from http://www.rcpsych.ac.uk/mentalhealthinfoforall/ problems/depression/antidepressants.aspx.

RxISK website (2017). https://rxisk.org/.

Samaha AN, Seeman P, Stewart J, Rajabi H, Kapur S (2007). 'Breakthrough' dopamine supersensitivity during ongoing antipsychotic treatment leads to treatment failure over time. Journal of Neuroscience 27, 2979-2986.

Schmeid K, Ernst K (1983). Isolierung and zwangsinjektion in urteil der betroffenen patienten und des pflegepersonals. Archiv fur Psychiatrie und Nervenkrankenheiten 233, 211-222.

Stahl SM (2013). Stahl's Essential Psychopharmacology, 4th edn. Cambridge University Press: Cambridge.

Vernon AC, Natesan S, Modo M, Kapur S (2011). Effect of chronic antipsychotic treatment on brain structure: a serial magnetic resonance imaging study with ex vivo and postmortem confirmation. Biological Psychiatry 69, 936-944.

Vinkers CH, Olivier B (2012). Mechanisms underlying tolerance after long-term benzodiazepine use: a future for subtype-selective GABA(A) receptor modulators? Advances in Pharmacological Science 2012, 416864. 